\title{
S. A. Tissot und sein Einfluss auf den «Dessau-Wörlitzer Kulturkreis»*
}

von Jürgen Konert

\section{ZuSAMMENFASSUNG}

Das kleine Fürstentum Anhalt-Dessau stellte in der zweiten Hälfte des 18. Jh.s einen aufgeklärten Musterstaat dar. Hier wurden auch vorbildliche Reformen im Medizinalwesen durchgeführt. Grundlegende Anregungen dafür kamen nachweislich aus der Schweiz, namentlich von S. A. Tissot (1728-1797), mit dem Fürst Leopold Friedrich Franz von Anhalt-Dessau (1740-1817) in persönlichem Kontakt stand. Tissots Einfluss ist besonders im schriftstellerischen Werk des Dessauer Arztes Samuel Friedrich Kretzschmar (1730-1793) und in der Einführung der Pockenschutzimpfung in ihrer ersten Form, der Variolation, zu erkennen.

In der Spätphase der Aufklärung spielte das kleine Fürstentum AnhaltDessau, an der Elbe etwa in der Mitte zwischen Halle/Leipzig und Berlin gelegen, eine Vorbildrolle.

Unter der Regierung des Fürsten Leopold Friedrich Franz (1740-1817) entstand in der 2. Hälfte des 18. Jahrhunderts ein kleiner Musterstaat. Dieser «Dessau-Wörlitzer Kulturkreis» - ein Begriff, der sich seit den zwanziger Jahren unseres Jahrhunderts in der Kulturgeschichte herausgebildet hat $^{1}$ - und der sich an den beiden Zentren, der Hauptstadt Dessau und dem Schlosspark Wörlitz, orientiert - umfasst aufgeklärte Leistungen, die von langanhaltender Nachwirkung waren. Kurzzeitig überstrahlten sie sogar den Ruhm von Weimar, auch wenn sie ausserhalb regionaler Grenzen heute kaum noch wahrgenommen werden. Im Zentrum stand dabei neben der Ökonomie, der Philosophie und der Gartenarchitektur besonders eine bürgerliche Bildungsreform. Die Leistungen auf dem Gebiet des Medizinalwesens wurden bisher jedoch zu wenig beachtet. Der 250.Geburtstag von Leopold Friedrich Franz war daher Anlass, mich der Frage zuzuwenden, ob

*) Vortrag, gehalten an der Jahrestagung der Schweiz. Gesellsch. f. Geschichte der Medizin und der Naturwissenschaften, 5./6. Okt. 1990 in Genf. 
auch in der Medizin in Anhalt der Einfluss der Aufklärung nachweisbar ist. Bei den hierzu erforderlichen Studien fanden sich sowohl Belege für persönliche Kontakte zwischen dem Dessauer Landesherren und dem bedeutenden Schweizer Arzt S. A. Tissot ${ }^{2}$ als auch für eine intensive Rezeption Tissotscher Werke und deren praktische Umsetzung in Mitteleuropa.

Repräsentant des Dessauer Medizinalwesens war der 1765 in das Fürstentum gekommene S.F. Kretzschmar (1730-1793). Er verkörperte den typischen Vertreter der medizinischen Aufklärung und trug wesentlich zur Umgestaltung des bis zu diesem Zeitpunkt schlecht entwickelten Medizinalwesens in Anhalt-Dessau bei ${ }^{3}$. Er verfasste mehrere medizinische Schriften, die Einblicke in das anhaltinische Medizinalwesen ermöglichen ${ }^{4}$. Von besonderer Bedeutung für unsere Fragestellung ist ein Vergleich seines 1768 erstmals erschienenen Buches «Irrthümer, Warnungen und Lehren, welche das Publikum in Ansehung der praktischen Arzneykunst betreffen» mit der 7 Jahre zuvor erschienenen Schrift von Tissot «Avis au peuple sur sa santé». Beide Werke erfreuten sich grosser Beliebtheit. So wurde das Buch von Tissot in zahlreiche Sprachen übersetzt, und dasjenige von S. F. Kretzschmar fand bereits zwei Jahre später in Würzburg eine zweite Auflage. In ihren Zielen und im Adressatenkreis stimmten sie weitgehend überein, waren sie doch ursprünglich beide für das begrenzte umliegende Territorium und die dort ansässige einfache Bevölkerung konzipiert. Angestrebt wurde neben einer intellektuellen Aufklärung besonders die praktische Erziehung zu einer gesunden Lebensweise. Die Ähnlichkeiten beider Publikationen beschränken sich dabei nicht nur auf konzeptionelle Fragen, sondern wurden auch im Inhalt sichtbar. Deutlich ist die Kretzschmarsche Schrift über zeittypische Gestaltungskonzepte hinaus vom Tissotschen Werk geprägt. Kretzschmar selbst bestätigt das auch in seiner Vorrede, wenn er die Schrift von Tissot als besonders wertvoll für die Bevölkerungsaufklärung hervorhebt $^{5}$. Er ist der Ansicht, wenn mehr derartige Schriften in die Hände der Bürger gelangten, dann könnten in absehbarer Zeit gefährliche Irrtümer und der verhängnisvolle Aberglaube abgebaut werden. In beiden Abhandlungen nimmt der Kampf gegen das vor allem auf dem Lande weit verbreitete medizinische Pfuschertum eine Zentralstellung ein. Tissot widmet mehrere Kapitel dem Thema «Von den Marktschreyern und Quacksalbern» und den «falschen Dorfärzten»:

«Ich muss noch von einer Plage reden, die mehr Verwüstung als alle anderen Übel anrichtet, und die, solange sie fortdauert, alle Sorgfalt für die Erhaltung des gemeinen Mannes 
fruchtlos macht, ich meine die Marktschreyer [...] Entblösst von allen Einsichten und von allen Erfahrungen, mit drey oder vier gemeinigliche gewaltsamen Mitteln bewaffnet, deren Beschaffenheit sie eben so wenig als die Krankheit kennen, für die sie verordnet wurden, sind sie gleich einem Rasenden, der ein Schwert in der Hand führt ${ }^{6} . »$

Auch S. F. Kretzschmar ist überzeugt, dass Marktschreier, Scharlatane und «Afterärzte» eine ernstliche Gefahr für die Bevölkerung darstellen. Da sie

«durch ein hirnloses Geschwätze von der Erkenntniss der Krankheiten und ihrer Ursachen aus dem Urine, von dem Antun, Behexen und Beschreyen, von den Besitzungen und von den Wirkungen des Teufels bey dieser und jener Krankheit Einfluss erlangen».

Das Volk lässt sich durch die Narrenpossen der Marktschreier blenden; Universalmittel, verbunden mit Behexung und Teufelsaustreibung wurden den Scharlatanen auf den Märkten meist teuer abgekauft. Die Heilung der Krankheiten beruht nach Kretzschmar nicht nur auf der Anwendung eines bestimmten Mittels, sondern auch auf der Wissenschaft und Ausbildung der Ärzte. Diese Wissenschaft kann z. B. «ein Afterarzt, ein Reffträger, eine Frau Nachbarin» ${ }^{4}$ nicht besitzen.

Auch im praktischen Teil der Schriften sind die Ähnlichkeiten wohl nicht zufällig. Beide Autoren erklären die einzelnen Krankheiten und ihre Symptome ausführlich und gut verständlich. Es schliessen sich Hinweise zur Therapie an. Die Publikation von Kretzschmar widerspiegelt eindeutig die Akzeptanz des Tissotschen Werkes im mitteldeutschen Raum, schon wenige Jahre nach seinem Erscheinen.

Analysiert man das geistige Umfeld in Dessau, so verwundert diese schnelle Rezeption nicht. Die Beziehung zur Schweizer Medizin, die im 18. Jahrhundert einen Höhepunkt ihrer Entwicklung erlebte, lässt sich bei Kretzschmar weiter zurückverfolgen. So wurde er von Johann Ulrich Bilguer (1720-1796) nach Dessau empfohlen. Der bedeutende Feldchirurg Friedrichs II. war ein gebürtiger Schweizer und hatte nach Beendigung des Siebenjährigen Krieges 1764 seine in Halle verteidigte Dissertation über Amputationsindikationen in Lausanne drucken lassen ${ }^{7}$. Er war ein Freund Tissots und begründete den Kontakt Kretzschmars mit dem Gedanken der medizinischen Aufklärung.

In Dessau wurde die Beschäftigung mit den Werken der Schweizer Medizin und vor allem Tissots noch durch die Gemahlin des Fürsten Franz, Luise (1750-1811), gefördert. Sie stand in enger brieflicher Verbindung mit dem schweizerischen Hofarzt in Hannover, Johann Georg Zimmermann $(1728-1795)^{8}$. Er war ein Schüler von Albrecht von Haller (1708-1777) und 
ein enger Freund Tissots. Auf Anregung von Kretzschmar fand 1770 auch die Reise des Fürstenpaares nach Lausanne statt, wo es im Juli zum Treffen mit Tissot kam. Zu diesem Zeitpunkt galt dieser somit in Dessau bereits als eine bedeutende ärztliche Persönlichkeit. Tissot vermittelte dem interessierten Fürsten wohl nicht nur Anregungen für seine gesunde Lebensweise und späteren Bäderreisen, vor allem nach England, sondern auch für konkrete gesundheitspolitische Massnahmen innerhalb seines kleinen Landes.

Die eingehende Beschäftigung mit der Volksgesundheit und der öffentlichen Hygiene war ein Thema, das in der 2. Hälfte des 18. Jahrhunderts gewissermassen in der Luft lag und nach Bearbeitung rief. Die Idee der Aufklärung und insbesondere J. J. Rousseau hatten das Terrain hinlänglich vorbereitet, ja geradezu das Bedürfnis nach einer solchen medizinischen Betrachtung geschaffen. Es ist vor diesem Hintergrund verständlich, dass man in Dessau dankbar jede Anregung aufgriff und sie nach Kräften weiterentwickelte. Zahlreiche Beispiele belegen den Schweizer Einfluss.

An erster Stelle sei auf die Einführung der Pockenimpfung hingewiesen, die zu diesem Zeitpunkt noch nicht allgemein üblich war. Sie wurde 1748 erstmals auf dem Kontinent durch den Schweizer Arzt Théodore Tronchin (1709-1781) durchgeführt und fand ihre publizistische Unterstützung in Tissots Schrift «L'inoculation justifiée» aus dem Jahre 1754. Diese Form der Pockenvorbeugung durch Einimpfung der «Blattern» war jedoch nicht ungefährlich und rief zahlreichen Widerspruch hervor. Dennoch liess der Fürst Franz nicht nur seinen eigenen Sohn impfen, sondern machte diese Massnahme zur Voraussetzung für eine Aufnahme in die bekannte Schuleinrichtung Philanthropinum ${ }^{1}$.

Eine der Hauptleistungen der Dessauer Aufklärung stellt die Verwirklichung der bürgerlichen Schulreform dar. Sie ist höchster Ausdruck der in die Tat umgesetzten Philosophie der Aufklärung, die u. a. die Trennung des Schulwesens von der Kirche forderte. Mit dem vom J.B. Basedow (17241790) begründeten Philantropinum wurde eine «Pädagogische Kolonie» geschaffen, in der auch auf medizinischem Gebiet Fortschrittliches geleistet wurde. In vielen Details bildeten Philantropismus und Gesundheitspolitik eine Einheit. Kretzschmar konnte dabei als Lehrer an dieser Bildungseinrichtung direkt gesundheitserzieherisch auf die Jugend einwirken. Er wurde somit zu einem «Ahnherrn der deutschen Schulärzte».

Neben hygienischen Fragen galt seine besondere Aufmerksamkeit der Erziehung zu einer bewussten Lebensführung und der Propagierung körperlicher Betätigung zur Erhaltung und Förderung der Gesundheit. Ausdruck 
hierfür sind die Rolle der «Turnkunst» im Unterricht und die sportlichen Wettkämpfe beim Drehbergfest ${ }^{9}$.

Kretzschmars Bestrebungen aber gingen weit darüber hinaus, und als er 1772 Leopold Friedrich Franz seine Pläne für eine grundsätzliche Medizinalreform vorlegte, war dieser den Gedanken seines Leibarztes gegenüber sehr aufgeschlossen. Die durch eine flexible Steuerpolitik aufblühende Ökonomie des kleinen Ländchens ermöglichte die erforderliche Mittelbereitstellung. Die durchgeführte Reglementierung (Zulassungskontrolle, Einteilung in Physicatdistricte usw.) brachte eine spürbare Verbesserung auf humanitärem Gebiet und entspricht voll der Umsetzung aufgeklärter "polizeilicher» Sorge für den Bürger in der Praxis. Neben der Errichtung des ersten Krankenhauses in Dessau im Jahre 1786 stand die kostenlose ärztliche Versorgung der Armen durch dazu angestellte Ärzte und Wundärzte im Vordergrund.

Die Veränderungen auf dem Gebiet des Medizinalwesens, die nachweislich auf den Kontakt Kretzschmars und des Fürsten Franz mit Tissot und seinen Vorstellungen beruhten, widerspiegeln den Einfluss der Schweizer Medizin. Sie verwirklichten zahlreiche Forderungen der aufgeklärten Ärzte und machten Anhalt-Dessau auch auf diesem Gebiet zu einem seiner Zeit vorauseilenden Staat.

\section{Literatur}

1 Hirsch, Erhard, Dessau-Wörlitz, Aufklärung und Frühklassik, Leipzig 1985.

2 Olivier, E., Médecine et santé dans le pays de Vaud au 18* siècle, Lausanne 1959.

3 Völker, Arina, Die Entwicklung von Medizinalorganisation und Bevölkerungsversorgung am territorialen Beispiel von Anhalt, Halle 1985.

4 Kretzschmar, S. F., Nachrichten von der Versorgung der Armen im Fürstentum Anhalt-Dessau, Dessau 1789.

5 Kretzschmar, S. F., Irrthümer, Warnungen und Lehren, welche das Publicum in Ansehung der praktischen Arzneikunst betreffen, Würzburg, Bamberg, Fulda 1770.

6 Tissot, S.A.D., Anleitung für den gemeinen Mann in Absicht auf seine Gesundheit; oder gemeinnütziges und bewährtes Haus-Arzney-Buch, Mannheim, 1772.

7 Bilguer, Johann, Ulrich, Dissertation sur l'inutilité de l'amputation des membres, Lausanne 1764. Originalfassung: Dissertatio de membrorum amputatione rarissime administranda aut quasi abroganda, Halle 1761. Die aufsehenerregende Schrift wurde auch ins Deutsche, Englische, Holländische und Spanische übersetzt.

8 Wäschke, H., Aus klassischer Zeit. Briefe des Hofarztes Dr.Zimmermann an die Herzogin Luise, in Anhalt. Geschichtsbl. 1, 1925, p. 127.

9 Lukas, G., Sportliche Feste in Wörlitz des Leopold Friedrich Franz von Anhalt Dessau, in Dessau Kultur, Dessau 1966. 


\section{Summary}

\section{Tissot's influence in Anhalt-Dessau}

In the 2nd half of the 18th century, the small Duchy of Anhalt-Dessau was the model of an enlightened State, also in matters of health policy. The author stresses the influence of the Swiss Samuel-André Tissot on both Prince Leopold Friedrich Franz (1740-1817) and his foremost physician, Samuel Friedrich Kretzsehmar (1730-1793). Tissot's influence manifested itself also in the early introduction of variolation.

\section{Résumé}

\section{L'influence de Tissot dans la Principauté d'Anhalt-Dessau}

La petite principauté d'Anhalt-Dessau offrait dans la $2^{\mathrm{e}}$ moitié du $18^{\mathrm{e}}$ siècle le modèle d'un état éclairé. C'est dans ce cadre que furent également conduites des réformes exemplaires dans le domaine de la médecine. Des impulsions fondamentales en vinrent de la Suisse. Le point de départ fut constitué par les contacts personnels entre S.A.Tissot (1728-1797) et le prince Leopold Friedrich Franz von Anhalt-Dessau (1740-1817). A côté de nombreuses publications médicales en relation avec les travaux de Tissot, il faut signaler avant tout à cet égard l'introduction rapide à Dessau de la vaccination contre la petite vérole qui avait été inaugurée , sur le continent, par Th. Tronchin (1709-1781) et soutenue par Tissot.

OA Dr.sc. med. Dr. phil. J. Konert

MLU Halle-Wittenberg

Bereich Medizin

Abt. Ethik und Geschichte der Medizin

Krausenstrasse 14

D-O-4020 Halle/S. 